\title{
Impact of the Early Phase of the COVID-19 Pandemic on US Healthcare Workers: Results from the HERO Registry
}

\author{
Christopher B. Forrest, MD, $P h D^{7}$ (D) , Haolin Xu, MS ${ }^{2}$, Laine E. Thomas, $P h D^{2}$, \\ Laura E. Webb, $B S^{2}$, Lauren W. Cohen, $M A^{2}$, Timothy S. Carey, MD, $M P H^{3}$, \\ Cynthia H. Chuang, MD, MSC ${ }^{4}$, Nancy M. Daraiseh, $P h D^{5}$, Rainu Kaushal, $M D^{6}$, \\ James C. McClay, MD, MS $S^{7}$, François Modave, $P h D^{8}$, Elizabeth Nauman, $M P H, P h D^{9}$, \\ Jonathan V. Todd, PhD, MSPH ${ }^{10}$, Amisha Wallia, MD, MS ${ }^{17}$, Cortney Bruno, MSW', \\ Adrian F. Hernandez, MD, MHS' ${ }^{2}$, and Emily C. O'Brien, PhD'2for the HERO Registry \\ Research Group
}

\begin{abstract}
${ }^{1}$ Applied Clinical Research Center, Children's Hospital of Philadelphia, Philadelphia, PA, USA; ${ }^{2}$ Duke Clinical Research Institute, Duke University School of Medicine, Durham, NC, USA; ${ }^{3}$ Department of Medicine, University of North Carolina at Chapel Hill, Chapel Hill, NC, USA; ${ }^{4}$ Department of Medicine, Penn State College of Medicine, Hershey, PA, USA; ${ }^{5}$ Cincinnati Children's Hospital, University of Cincinnati, Cincinnati, OH, USA;

${ }^{6}$ Department of Population Health Sciences, Weill Cornell Medicine, New York, NY, USA; ${ }^{7}$ University of Nebraska Medical Center, Omaha, NE, USA; ${ }^{8}$ Department of Health Outcomes and Biomedical Informatics, College of Medicine, University of Florida, Gainesville, FL, USA; ${ }^{9}$ Louisiana Public Health Institute, New Orleans, LA, USA; ${ }^{10} \mathrm{OCHIN}$, Inc., Portland, OR, USA; " Division of Endocrinology, Metabolism and Molecular Medicine,

Department of Medicine and the Institute for Public Health and Medicine, Northwestern University Feinberg School of Medicine, Chicago, IL, USA.
\end{abstract}

BACKGROUND: The HERO registry was established to support research on the impact of the COVID-19 pandemic on US healthcare workers.

OBJECTIVE: Describe the COVID-19 pandemic experiences of and effects on individuals participating in the HERO registry.

DESIGN: Cross-sectional, self-administered registry enrollment survey conducted from April 10 to July 31, 2020. SETTING: Participants worked in hospitals (74.4\%), outpatient clinics (7.4\%), and other settings (18.2\%) located throughout the nation.

PARTICIPANTS: A total of 14,600 healthcare workers.

MAIN MEASURES: COVID-19 exposure, viral and antibody testing, diagnosis of COVID-19, job burnout, and physical and emotional distress.

KEY RESULTS: Mean age was 42.0 years, $76.4 \%$ were female, $78.9 \%$ were White, $33.2 \%$ were nurses, $18.4 \%$ were physicians, and $30.3 \%$ worked in settings at high risk for COVID-19 exposure (e.g., ICUs, EDs, COVID-19 units). Overall, 43.7\% reported a COVID-19 exposure and $91.3 \%$ were exposed at work. Just 3.8\% in both high- and low-risk settings experienced COVID-19 illness. In regression analyses controlling for demographics, professional role, and work setting, the risk of COVID-19 illness was higher for Black/African-Americans (aOR 2.32, 99\% CI 1.45, 3.70, $p<0.01$ ) and Hispanic/Latinos (aOR 2.19, $99 \%$ CI 1.55, 3.08, $p<0.01$ ) compared with Whites. Overall, $41 \%$ responded that they were experiencing job burnout. Responding about the day before they completed the survey, 53\% of participants reported feeling tired a lot of the day, $51 \%$ stress, $41 \%$ trouble sleeping, 38\% worry,

Please see Appendix for list of HERO Registry Research Group collaborators.

Received August 18, 2020

Accepted December 20, 2020

Published online March 10, 2021
$21 \%$ sadness, 19\% physical pain, and 15\% anger. On average, healthcare workers reported experiencing 2.4 of these 7 distress feelings a lot of the day.

CONCLUSIONS: Healthcare workers are at high risk for COVID-19 exposure, but rates of COVID-19 illness were low. The greater risk of COVID-19 infection among race/ ethnicity minorities reported in the general population is also seen in healthcare workers. The HERO registry will continue to monitor changes in healthcare worker wellbeing during the pandemic.

TRIAL REGISTRATION: ClinicalTrials.gov identifier NCT04342806

KEY WORDS: COVID-19; SARS-CoV-2; healthcare worker; registry; disparities; burnout; well-being.

J Gen Intern Med 36(5):1319-26

DOI: $10.1007 / \mathrm{s} 11606-020-06529-\mathrm{Z}$

(c) Society of General Internal Medicine 2021

\section{INTRODUCTION}

The 18 million people in the US healthcare workforce ${ }^{1}$ are essential to the nation's pandemic response. Many are at risk for exposure to an infected person at work, and a substantial portion may experience severe illness if infected. ${ }^{2}$ The pandemic has placed exceptional organizational and financial strain on healthcare institutions. ${ }^{3,}{ }^{4}$ Physical and emotional symptoms due to the demands of the pandemic appear to be common among healthcare workers in international settings. 5 , 6

Much of the evidence on the impact of the pandemic on healthcare workers, including infection rates, risk factors, and well-being, comes from international studies ${ }^{7}$ or US studies limited to single hospitals or health systems. ${ }^{8-10}$ We lack 
essential information about all healthcare workers' experiences with the pandemic, not just those infected with SARS$\mathrm{CoV}-2$ or those with direct patient care responsibilities. There also is a need for data from large-scale US studies to better describe how the pandemic is affecting US healthcare workers' personal and job-related well-being, ${ }^{11}$ exposure and infection rates, and whether the racial and ethnic disparities in risk of SARS-CoV-2 infection seen in the general popula$\operatorname{tion}^{12-15}$ are also present in US healthcare workers.

To begin filling these gaps, we launched the Healthcare Worker Exposure Response and Outcomes (HERO) research program (heroesresearch.org) in April 2020. Individual healthcare workers were invited to join the program by enrolling in a registry. Any adult who worked in a healthcare facility that delivered direct patient care was eligible to join. In this manuscript, we present initial results from the HERO registry enrollment survey of 14,600 healthcare workers who joined from April to July 2020. Our objectives are to describe in a national, convenience sample of healthcare workers the baseline characteristics of exposures to COVID-19; viral and antibody testing; physician diagnosis of COVID-19; job burnout; and, physical and emotional distress experiences. We contrast these experiences between personnel who work in settings at high risk for exposure (e.g., emergency departments and intensive care units) with those in lower risk settings.

\section{METHODS}

\section{HERO Registry}

Established in April 2020 with funding from the PatientCentered Outcomes Research Institute, the HERO research program is one of the research activities of PCORnet ${ }^{\circledR}$, the National Patient-Centered Clinical Research Network (pcornet.org) ${ }^{16}$ At its launch, the HERO research program included a registry (ClinicalTrials.gov Identifier NCT04342806), data from which this manuscript reports, and a clinical trial on prophylactic use of hydroxychloroquine (ClinicalTrials.gov Identifier NCT04334148). The clinical trial activated 40 recruitment sites, which were the primary sources of registry recruitment as well. All recruitment sites were part of or closely affiliated with academic medical centers.

Study procedures were reviewed by the Duke University School of Medicine Institutional Review Board and approved by the Western Institutional Review Board (Pro00105284). The registry defined a healthcare worker as anyone (e.g., clinicians, support staff, technicians, administrators, etc.) who works in a facility or organization that provides healthcare services to patients (e.g., hospitals, emergency medical services, clinics, skilled nursing facilities, first responders). Registry members had to be 18 years or older and able to read English. Within health systems participating in PCORnet, ${ }^{16}$ recruitment was done via advertisements, institution-wide emails, provider recommendations, and newsletters. Additional approaches included articles in the media, social media advertisement, and professional society solicitations. Individuals accessed a website (heroesresearch. org) to join. None of these approaches was based on a denominator of eligible participants, as any adult healthcare worker in the nation was potentially eligible. Thus, we were unable to compute response rates for this cross-sectional survey.

Participants enrolled from April 10, 2020, to July 31, 2020. After electronically signing the consent form, they created a profile with contact information, demographics, employment characteristics, and interest in participating in future studies. The enrollment survey also contained questions about exposures, viral and antibody testing, COVID-19 illness history, symptoms, and job burnout.

\section{Demographic and Work-Related Variables}

Demographics included age, gender, race, ethnicity, and state of residence. We combined race and ethnicity into a single variable by assigning individuals to Hispanic/Latino if they endorsed this category in the ethnicity question; otherwise, we used their self-assigned race as Black/African-American, White, Asian/Pacific Islander, or Other. Participants indicated the type of healthcare facility where they were employed and their professional role. We used this information to construct a COVID-19 work setting exposure risk variable. High-risk settings included intensive care units, emergency departments/urgent care centers, COVID-19 therapeutic or diagnostic units, respiratory services, anesthesia, and emergency medical services. Individuals who reported that one of these settings was their primary place of work, regardless of their role, were assigned to the high-risk category; other settings were considered low risk.

\section{COVID-19 Exposures, Testing, and Illness}

We asked healthcare workers if they believed they had been exposed to an individual with COVID-19 illness, either at work or in their communities, and whether they had undergone viral or antibody testing, and if so, the result(s). Because access to viral testing was limited in the early months of the pandemic, we also asked if they had been diagnosed with COVID-19 without undergoing viral testing.

\section{Job Burnout}

We used a previously developed single item that has been shown to be a valid indicator of job burnout. ${ }^{17}$ Participants are instructed to define burnout for themselves: "Overall, based on your definition of burnout, how would you rate your level of burnout?" Responses are scored on a five-category ordinal scale, where 1 = "I enjoy my work. I have no symptoms of burnout"; 2 = "Occasionally I am under stress, and I don't always have as much energy as I once did, but I don't feel burned out"; 3 = "I am definitely burning out and have one or more symptoms of burnout, such as physical and emotional 


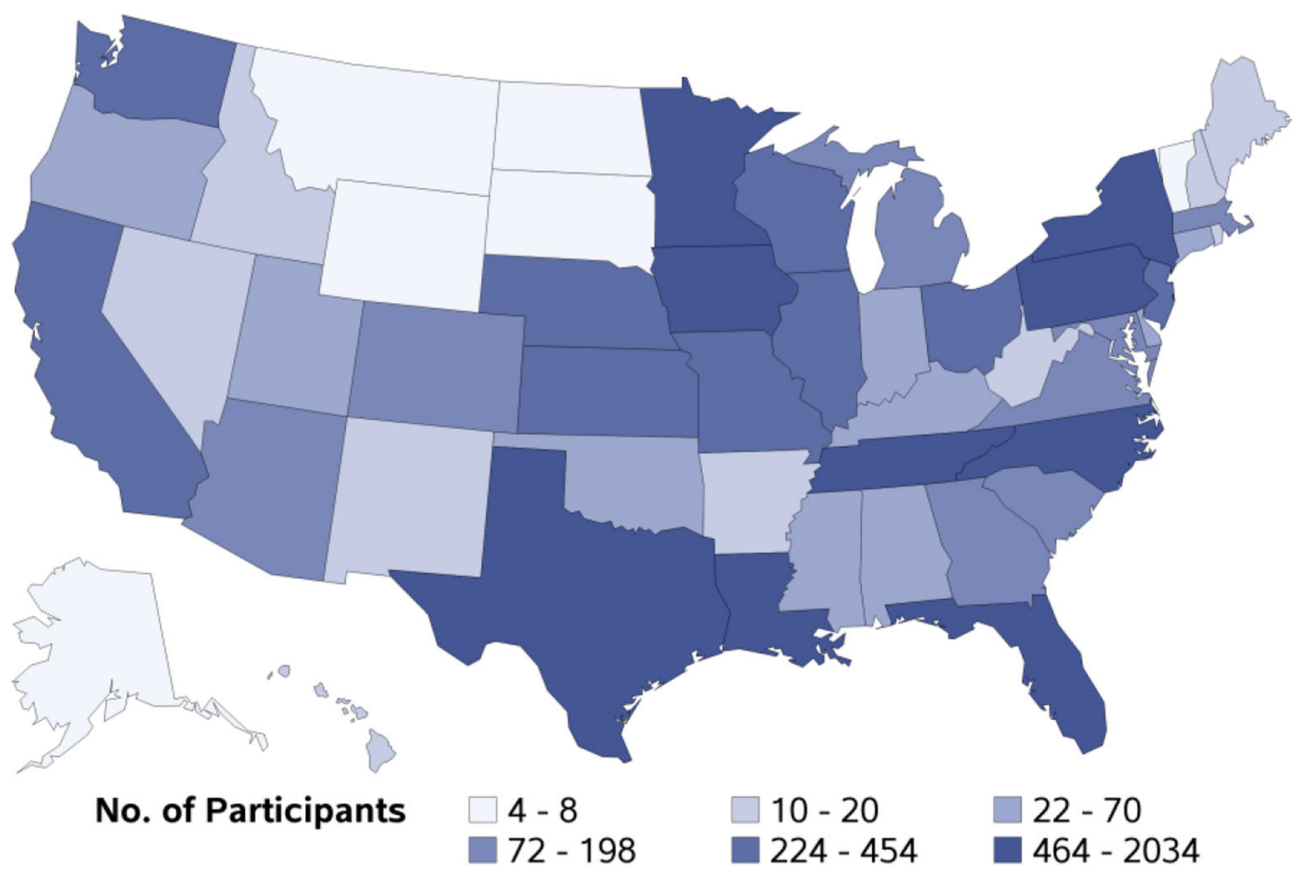

Figure 1 State of residence for 14,600 healthcare workers enrolled in the HERO registry. The map shows the count of healthcare workers who enrolled in the HERO research community via a web-based registry. Recruitment methods included communications done within healthcare systems that participate in PCORnet (pcornet.org), a national network-of-networks devoted to conducting clinical research; media articles and televised stories; social media; and professional societies. The count of the number of participants by state is based on individuals' recorded 5digit zip codes of residence.

exhaustion"; 4 = "The symptoms of burnout that I'm experiencing won't go away. I think about frustration at work a lot"; and 5 = "I feel completely burned out and often wonder if I can go on. I am at the point where I may need some changes or may need to seek some sort of help." Consistent with prior studies, ${ }^{17,}{ }^{18}$ we dichotomized the item as $\leq 2$ (no burnout) vs. $\geq 3$ (job burnout).

\section{Physical and Emotional Distress Experiences}

We used a 1-day recall period to capture negative affective daily life experiences, which people have difficulty remembering accurately over longer periods of time. ${ }^{19}$ This approach is based on ecological momentary assessment, which samples individuals' experiences in near-real time to minimize recall bias and maximize ecological validity. ${ }^{20}$ By using the phrase "a lot of the day," we added a high level of symptom severity to each item while maintaining the efficiency of a yes/no question. The questions we developed were based on a modification of the Gallup negative experiences index, which is used in the annual Global Well-Being report. ${ }^{21}$ Participants were asked about physical (trouble sleeping, pain, and fatigue) and emotional (worry, sadness, anger, and stress) distress experienced "yesterday." Each question used a yes/no response option. Except for the trouble sleeping item, which asked about last night, the other items were worded as "Did you feel [insert feeling] a lot of the day yesterday?"

\section{Statistical Analyses}

We used 5-digit zip code of residence to obtain a count of participants per state and then used those counts to create a national heat map of HERO participants. In bivariate analyses, we contrasted individuals whose work setting was high versus low risk for COVID-19 exposure; contrasts included demographics, type of facility where employed, professional role, COVID-19 testing and infection experiences, job burnout, and daily physical and emotional symptoms. We computed unadjusted proportions for viral testing or COVID-19 illness rates overall and by week of survey completion and race/ethnicity category. Statistical significance was evaluated using chi-squared tests for proportions and linear trends and $t$ tests for means.

We fit three multivariable logistic regression models to explore associations for three outcomes: (1) COVID19 infection (yes/no) as determined by a positive viral test, positive antibody test, or physician diagnosis; (2) job burnout (yes/no); and (3) count of the number of daily distress experiences. The first two regressions used a logit link and the third a Poisson link function. The explanatory variables included age, gender, race/ethnicity, type of healthcare facility where employed, job role, work setting risk for COVID-19 exposure, week of survey completion, and geographic region of residence. Results are reported as adjusted odds ratios (aORs) with 99\% confidence limits for the 
Table 1 Healthcare Worker Characteristics by the COVID-19 Exposure Risk of Their Work Setting

\begin{tabular}{|c|c|c|c|c|}
\hline \multirow[b]{2}{*}{ Characteristic } & \multirow[b]{2}{*}{ Overall } & \multicolumn{2}{|c|}{ Work setting exposure risk* } & \multirow[b]{2}{*}{$p$ value } \\
\hline & & High & Low & \\
\hline & $N=14,600$ & $N=4423$ & $N=10,177$ & \\
\hline Age, mean (SD), years & $42.0(11.5)$ & $39.6(10.7)$ & $43.0(11.6)$ & $<0.01$ \\
\hline \multicolumn{4}{|l|}{ Age group, $n(\%)$, years } & $<0.01$ \\
\hline $\begin{array}{l}18-29 \\
30-49\end{array}$ & $2114(14.5)$ & $813(18.4)$ & $1301(12.8)$ & \\
\hline $\begin{array}{l}30-49 \\
50-64\end{array}$ & $8566(58.7)$ & $2729(61.7)$ & $5837(57.4)$ & \\
\hline $50-64$ & $3578(24.5)$ & $830(18.8)$ & $2748(27.0)$ & \\
\hline $65+$ & $342(2.3)$ & $51(1.2)$ & $291(2.9)$ & \\
\hline Female, $n / \%$ & $11,149(76.4)$ & $3188(72.1)$ & $7961(78.2)$ & $<0.01$ \\
\hline \multicolumn{4}{|l|}{ Race/ethnicity, $n(\%)$} & 0.04 \\
\hline Hispanic/Latino (any race) & $1128(7.7)$ & $302(6.8)$ & $826(8.1)$ & \\
\hline Black/African-American & $527(3.6)$ & $147(3.3)$ & $380(3.7)$ & \\
\hline White & $11,523(78.9)$ & $3523(79.7)$ & $8000(78.6)$ & \\
\hline Asian/Pacific Islander & $917(6.3)$ & $291(6.6)$ & $626(6.2)$ & \\
\hline Other (include other race, mixed) & $505(3.5)$ & $160(3.6)$ & $345(3.4)$ & \\
\hline \multicolumn{4}{|l|}{ Type of healthcare facility, $n(\%)$} & $<0.01$ \\
\hline Hospital & $10,864(74.4)$ & $3876(87.6)$ & $6988(68.7)$ & \\
\hline Outpatient clinic/facility & $1083(7.4)$ & $26(0.6)$ & $1057(10.4)$ & \\
\hline Skilled nursing facility & 275 (1.9) & $22(0.5)$ & $253(2.5)$ & \\
\hline Emergency services & $242(1.7)$ & $200(4.5)$ & $42(0.4)$ & \\
\hline Other & 2136 (14.6) & $299(6.8)$ & $1837(18.1)$ & \\
\hline Professional role, $n(\%)$ & & & & $<0.01$ \\
\hline \multicolumn{4}{|l|}{ Clinical } & \\
\hline Nurse (RN/LPN) & $4776(33.2)$ & $1939(44.2)$ & $2837(28.4)$ & \\
\hline Physician & 2645 (18.4) & $711(16.2)$ & $1934(19.3)$ & \\
\hline Physician-in-training & $473(3.3)$ & $146(3.3)$ & $327(3.3)$ & \\
\hline Paramedic/emergency medical technician & $412(2.9)$ & $409(9.3)$ & $3(0.0)$ & \\
\hline Physician's assistant (PA) & $372(2.6)$ & $121(2.8)$ & $251(2.5)$ & \\
\hline Nurse practitioner (NP) & $728(5.1)$ & $142(3.2)$ & $586(5.9)$ & \\
\hline Respiratory therapist & $225(1.6)$ & $206(4.7)$ & $19(0.2)$ & \\
\hline Physical therapist (PT) & $248(1.7)$ & $30(0.7)$ & $218(2.2)$ & \\
\hline Medical assistant & $163(1.1)$ & $30(0.7)$ & $133(1.3)$ & \\
\hline \multicolumn{5}{|l|}{ Non-clinical } \\
\hline Administrative staff & $706(4.9)$ & $93(2.1)$ & $613(6.1)$ & \\
\hline Environmental services & $78(0.5)$ & $17(0.4)$ & $61(0.6)$ & \\
\hline Laboratory technician & $208(1.4)$ & $7(0.2)$ & $201(2.0)$ & \\
\hline Pharmacist/pharmacy technician & $427(3.0)$ & $64(1.5)$ & $363(3.6)$ & \\
\hline Dietary/nutrition/food services & $98(0.7)$ & $8(0.2)$ & $90(0.9)$ & \\
\hline Other & 2,819 (19.6) & $459(10.5)$ & $2,360(23.6)$ & \\
\hline Missing & $222(1.5)$ & $41(0.9)$ & $181(1.8)$ & \\
\hline
\end{tabular}

*High COVID-19 exposure risk work settings included intensive care units, emergency departments/urgent care centers, COVID-19 therapeutic or diagnostic units, respiratory services, anesthesia, and emergency medical services. All other settings were considered low risk

logistic regressions, and adjusted rate ratios (aRRs) with 99\% confidence limits for the Poisson regression.

Analyses were done using SAS version 9.4 (SAS Institute Inc., Cary, NC, USA). $p$ values are 2-sided tests and were considered statistically significant at $<0.01$ because of the large sample size.

\section{RESULTS}

\section{Study Sample}

From April 10, 2020, to July 31, 2020, 14,600 healthcare workers joined the HERO registry, 8578 (58.8\%) enrolled in April, 3769 (25.8\%) in May, 1414 (9.7\%) in June, and $839(5.7 \%)$ in July. Individuals resided in all 50 states or the District of Columbia (Fig. 1). States with the largest numbers were Florida $(N=2034)$, New York $(N=1902)$, Pennsylvania $(N=1360)$, North Carolina $(N=1262)$, Tennessee $(N=1034)$, Minnesota $(N=960)$, Louisiana $(N=656)$, and Iowa $(N=607)$.
The mean participant age was 42.0 years, $76.4 \%$ were female, $78.9 \%$ were White, $74.4 \%$ worked in hospitals, and $33.2 \%$ were nurses (Table 1). High-risk settings were associated with younger workers and more males, hospital workers, and nurses compared with lower risk settings. Race/ethnicity did not differ by risk category. The professional roles reported were clinical (68.4\%), non-clinical (10.5\%), other (19.2\%), and missing (1.5\%).

\section{Testing and COVID-19 IIIness}

Overall, $43.7 \%$ of healthcare workers reported exposure to someone with COVID-19, and $91.3 \%$ of those reported an exposure at work (Table 2). Healthcare workers in high-risk settings were twice as likely to report an exposure at work, while low-risk healthcare workers were twice as likely to report being exposed in community settings. Exposure risk did not vary by week of enrollment across the study period $(p=0.53$, chisquared test for linear trend). 
Table 2 SARS-CoV-2 Exposures and Testing and COVID-19 Illness Among Healthcare Workers by the COVID-19 Exposure Risk of Their Work Setting

\begin{tabular}{|c|c|c|c|c|}
\hline \multirow[b]{2}{*}{ Characteristic } & \multirow[b]{2}{*}{ Overall } & \multicolumn{2}{|c|}{$\begin{array}{l}\text { Work setting exposure } \\
\text { risk }\end{array}$} & \multirow[b]{2}{*}{$p$ value } \\
\hline & & High & Low & \\
\hline & $N=14,600$ & $N=4423$ & $N=10,177$ & \\
\hline \multicolumn{5}{|l|}{ Exposure to SARS-CoV-2 virus, $n(\%)$} \\
\hline Any exposure & $6379(43.7)$ & $2895(65.5)$ & $3484(34.2)$ & $<0.01$ \\
\hline Exposed at work place & $5824(39.9)$ & $2817(63.7)$ & $3007(29.5)$ & $<0.01$ \\
\hline Exposed in community & $807(5.5)$ & $163(3.7)$ & $644(6.3)$ & $<0.01$ \\
\hline Exposed at work place among those with any exposure & $5824(91.3)$ & $2817(97.3)$ & $3007(86.3)$ & $<0.01$ \\
\hline \multicolumn{5}{|l|}{ SARS-CoV-2 viral test, $n(\%)$} \\
\hline Ever tested & $2460(16.8)$ & $806(18.2)$ & $1654(16.3)$ & $<0.01$ \\
\hline Tested positive & $268(1.8)$ & $88(2.0)$ & $180(1.8)$ & 0.36 \\
\hline Tested positive (among tested) & $268(10.9)$ & $88(10.9)$ & $180(10.9)$ & 0.98 \\
\hline \multicolumn{5}{|l|}{ SARS-CoV-2 antibody test, $n(\%)$} \\
\hline Ever tested & $1087(7.4)$ & $337(7.6)$ & $750(7.4)$ & 0.60 \\
\hline Tested positive & $126(0.9)$ & $38(0.9)$ & $88(0.9)$ & 0.97 \\
\hline Tested positive (among tested) & $126(11.6)$ & $38(11.3)$ & $88(11.7)$ & 0.83 \\
\hline Viral or antibody test positive, $n(\%)$ & $354(2.4)$ & $113(2.6)$ & $241(2.4)$ & 0.50 \\
\hline Physician-diagnosed COVID-19 illness without a viral test, $n(\%)$ & $235(1.6)$ & $62(1.4)$ & $173(1.7)$ & 0.19 \\
\hline SARS-CoV-2 viral or antibody test positive or physician diagnosis of COVID-19, $n(\%)$ & $554(3.8)$ & $164(3.7)$ & $390(3.8)$ & 0.72 \\
\hline
\end{tabular}

Despite their high rates of COVID-19 exposures, individuals in high-risk settings had similar rates of COVID-19 illness confirmed by viral testing, antibody testing, or diagnosis as those in low-risk settings. Among all healthcare workers, $2.4 \%$ had laboratory test evidence of SARS-CoV-2 infection (either viral or antibody test positive) and 3.8\% had either a positive test or physician diagnosis.

Viral testing was reported by $16.8 \%$ of participants, and antibody testing by $7.4 \%$. Among individuals who had a viral test, $10.9 \%$ had a positive result, and the test positivity rate did not differ by risk group. Similarly, among those who had an antibody test for SARS-CoV-2, $11.6 \%$ had a positive result with no difference between risk groups.

In logistic regression analyses in which COVID-19 illness was the outcome, there were no differences by age, sex, or work setting exposure risk (Table 3 ). The odds of COVID-19 illness was increased 2.32 times for Black/African-Americans and 2.19 times for Hispanic/ Latinos compared with Whites. The odds of COVID19 illness were reduced by $43 \%$ for individuals working in outpatient settings compared with those who worked in hospitals. Finally, the odds of infection increased by $4 \%$ each week during the study period.

\section{Burnout}

Overall, $41 \%$ of all healthcare workers responded that they were experiencing job burnout. In logistic regression analyses, job burnout was not associated with race/ethnicity, type of healthcare facility where employed, professional role, or week of survey administration (Table 3). Participants 50 years and older were less likely to report job burnout. The odds of reporting job burnout were 55\% higher for females than for males, and $31 \%$ higher for those who worked in high- versus low-risk settings.

\section{Distress Experiences}

Responding about the day before they completed the survey, $53 \%$ of participants reported feeling tired a lot of the day, $51 \%$ stress, $41 \%$ trouble sleeping, $38 \%$ worry, $21 \%$ sadness, $19 \%$ physical pain, and $15 \%$ anger. On average, healthcare workers reported 2.4 of a total of these 7 distress feelings a lot of the day. In Poisson regression analyses, the count of the number of negative distress feelings experienced the prior day decreased with age and week of survey completion and was higher for females and those who worked in high-risk settings (Table 3).

\section{DISCUSSION}

In this study of healthcare workers during the early phase of the pandemic (April-July 2020), 43.7\% reported exposure to someone with COVID-19 and $91.3 \%$ of those individuals were exposed at work. These high rates of exposure illustrate the safety risk that healthcare workers have faced in the conduct of their jobs. Despite high exposure rates, just 3.8\% reported a positive viral or antibody test or a diagnosis of COVID-19, a proportion that is consistent with other studies. ${ }^{7}$ That just 1 in 10 healthcare workers exposed to the virus had evidence of COVID-19 is good news. However, the risk of COVID-19 illness was not similar across race/ethnicity groups. We found that Black/African-American and Hispanic/Latino healthcare workers were twice as likely to contract COVID-19 as Whites, even after controlling for other demographic characteristics, including geographic region of residence, features of the work environment, and professional role. This study does not explain the reasons for these disparities, but it does add support to other researchers' calls to 
Table 3 Multivariable Regression Analyses for COVID-19 Illness, Job Burnout, and Daily Distress Experiences

\begin{tabular}{|c|c|c|c|}
\hline \multirow[t]{2}{*}{ Participant characteristic } & \multicolumn{3}{|l|}{ Outcome* } \\
\hline & COVID-19, aOR (99\% CI) & Burnout, aOR (99\% CI) & Distress experiences, aRR $(99 \% \mathrm{CI})$ \\
\hline \multicolumn{4}{|l|}{ Age, years } \\
\hline $18-29$ & Referent & Referent & Referent \\
\hline $30-49$ & $1.26(0.87,1.81)$ & $1.12(0.88,1.44)$ & $0.94(0.89,0.99)$ \\
\hline $50-64$ & $1.38(0.91,2.08)$ & $0.68(0.51,0.90)$ & $0.80(0.74,0.85)$ \\
\hline \multicolumn{4}{|l|}{ Gender } \\
\hline Male & Referent & Referent & Referent \\
\hline Female & $0.96(0.72,1.29)$ & $1.55(1.26,1.91)$ & $1.40(1.32,1.50)$ \\
\hline \multicolumn{4}{|l|}{ Race/ethnicity } \\
\hline White & Referent & Referent & Referent \\
\hline Black/African-American & $2.32(1.45,3.70)$ & $0.91(0.53,1.54)$ & $0.89(0.79,1.01)$ \\
\hline Hispanic/Latino & $2.19(1.55,3.08)$ & $0.95(0.69,1.32)$ & $1.02(0.95,1.10)$ \\
\hline Asian/Pacific Islander & $1.39(0.91,2.14)$ & $0.86(0.60,1.23)$ & $0.99(0.90,1.09)$ \\
\hline Other & $1.16(0.62,2.17)$ & $1.07(0.67,1.69)$ & $1.13(1.02,1.26)$ \\
\hline \multicolumn{4}{|l|}{ Type of healthcare facility } \\
\hline Hospital & Referent & Referent & Referent \\
\hline Outpatient clinic/facility & $0.57(0.33,0.99)$ & $1.06(0.74,1.50$ & $0.95(0.87,1.04)$ \\
\hline Skilled nursing facility & $0.97(0.41,2.31)$ & $2.05(1.00,4.22)$ & $1.25(1.09,1.43)$ \\
\hline Emergency services & $0.76(0.25,2.31)$ & $0.68(0.27,1.70)$ & $1.07(0.89,1.28)$ \\
\hline \multirow{2}{*}{\multicolumn{4}{|c|}{ Professional role }} \\
\hline & & & \\
\hline \multicolumn{4}{|l|}{ Clinical } \\
\hline Nurse & Referent & Referent & Referent \\
\hline Physician & $0.71(0.49,1.02)$ & $0.79(0.62,1.00)$ & $0.80(0.75,0.86)$ \\
\hline Physician-in-training & $0.77(0.40,1.48)$ & $0.68(0.42,1.09)$ & $0.83(0.73,0.95)$ \\
\hline Paramedic/EMT & $0.67(0.24,1.81)$ & $0.92(0.52,1.64)$ & $0.97(0.83,1.12)$ \\
\hline Physician's assistant & $0.96(0.48,1.90)$ & $0.97(0.60,1.57)$ & $0.82(0.72,0.94)$ \\
\hline Nurse practitioner & $1.18(0.69,2.01)$ & $0.85(0.60,1.21)$ & $0.99(0.91,1.09)$ \\
\hline Respiratory therapist & $0.95(0.36,2.51)$ & $0.65(0.32,1.30)$ & $0.97(0.82,1.14)$ \\
\hline Physical therapist & $1.42(0.67,2.99)$ & $0.96(0.55,1.70)$ & $0.91(0.78,1.07)$ \\
\hline Medical assistant & $1.27(0.46,3.45)$ & $1.50(0.56,4.01)$ & $1.07(0.89,1.28)$ \\
\hline \multicolumn{4}{|l|}{ Non-clinical } \\
\hline Administrative staff & $1.01(0.61,1.66)$ & $0.92(0.61,1.38)$ & $1.05(0.96,1.16)$ \\
\hline Environmental services & $\mathrm{n} / \mathrm{a}$ & $1.53(0.38,6.19)$ & $1.10(0.82,1.46)$ \\
\hline Laboratory technician & $0.54(0.16,1.79)$ & $1.55(0.80,3.01)$ & $1.03(0.86,1.22)$ \\
\hline Pharmacist & $0.29(0.09,0.97)$ & $1.20(0.74,1.95)$ & $0.91(0.80,1.03)$ \\
\hline Dietary/food services & $0.71(0.18,2.78)$ & $1.15(0.39,3.39)$ & $0.92(0.71,1.19)$ \\
\hline Other & $0.80(0.58,1.11)$ & $0.91(0.72,1.14)$ & $0.95(0.89,1.00)$ \\
\hline \multicolumn{4}{|l|}{ Work setting risk } \\
\hline Low & Referent & Referent & Referent \\
\hline High & $0.95(0.72,1.25)$ & $1.31(1.08,1.58)$ & $1.08(1.03,1.13)$ \\
\hline Week of survey completion & $1.04(1.01,1.07)$ & $1.01(0.95,1.07)$ & $0.98(0.97,0.99)$ \\
\hline
\end{tabular}

*The regression models controlled for type of healthcare facility where employed and geographic region of residence. Logistic regression was used for the COVID-19 illness and burnout outcomes, and Poisson regression for the distress experiences outcome. Italicized parameter estimates are significantly different from the referent at $p<0.01$. There were no cases of COVID-19 among environmental services workers

further examine and address COVID-19 race/ethnicity disparities. $^{12-15}$

This study relied on self-reported data for exposures and testing results, which can be affected by recall bias. Nonetheless, it is likely that healthcare workers have excellent knowledge of their testing history, the meaning of these tests, and good recall because of the profound impact of COVID-19. An important limitation of this study's external validity was the study sample selection. Although drawn from all 50 states and the District of Columbia, the sample was composed of volunteers who registered to be part of the HERO registry and should be considered a convenience sample. Participants were largely but not entirely recruited from within academic medical centers that participate in PCORnet. Figure 1 shows the national distribution of participants, but the sample cannot be considered nationally representative. Still, the study sample had demographic characteristics similar to an April 2020 CDC report of over 9000 healthcare workers infected with COVID19: median age was 42 years in both samples, $76 \%$ were female (vs $73 \%$ CDC sample), and $79 \%$ (vs 73\%) were White. ${ }^{22}$ This is also similar to the data from the US Census Bureau, which identified women holding $76 \%$ of health care jobs. $^{23}$

We found that $40.9 \%$ of our sample reported job burnout. In a 2015 study, the same measure was distributed to physicians, nurses, clinical associates, and administrative clerks - a diverse group of clinical and non-clinical healthcare workers similar to the sample in our study - in the Veterans Health Administration (VA), and investigators reported a job burnout rate of $38.5 \% .^{17}$ The similarity between our findings during the pandemic and those from the VA before the pandemic is striking. The HERO research program will continue to 
monitor job burnout to determine whether the pandemic has an impact on this important outcome over time.

Although the proportion of healthcare workers experiencing distress was high, the levels are not dissimilar from the US population. We contrasted the 2018 Gallup Emotions Survey $^{21}$ with our results. This contrast showed that the HERO registry sample had lower or the same proportions of pain, worry, sadness, stress, and anger as the US general population. This similarity reflects the resiliency of healthcare workers: despite the challenges and burdens of the pandemic, their distress experiences were comparable to the general population. Indeed, the level of distress decreased during the study period, which provides further evidence of the adaptive capacity of the healthcare workforce.

Our results suggest that additional research is needed to better understand how the pandemic is uniquely affecting female healthcare workers. After controlling for demographic and work characteristics, women were more likely than men to report job burnout and distress experiences. Our results do not reveal the origins of these differences or their consequences. The HERO research program is uniquely positioned to better understand changes in distress experiences over time and to conduct sub-studies that may illuminate causes for the genderbased differences, such as work-life balance.

This manuscript provides a broad description of the COVID-19 experiences and impact on US healthcare workers during the early phase of the pandemic (April-July 2020). It is a 4-month snapshot. The HERO program continues to enroll healthcare workers in its registry and intends to offer a variety of new studies to members. The registry can be used to monitor the impact of the pandemic over time and to better understand the differential impact it may be having on subpopulations, such as ethnic minorities and women.

Acknowledgments: The PCORnet ${ }^{\circledR}$ Study reported in this publication was conducted using PCORnet, the National Patient-Centered Clinical Research Network. PCORnet has been developed with funding from the Patient-Centered Outcomes Research Institute (PCORI). Please see the Appendix for the list of HERO Registry Research Group collaborators.

Corresponding Author: Christopher B. Forrest, MD, PhD; Applied Clinical Research Center, Children's Hospital of Philadelphia, Philadelphia, PA, USA (e-mail: forrestc@chop.edu).

Supplementary Information The online version contains supplementary material available at (https://doi.org/10.1007/s11606-02006529-z).

Funding The study was funded by PCORI through PCORI Award (COVID-19-2020-001), "HERO Registry \& Trial: Healthcare Worker Exposure Response and Outcomes."

\section{Compliance with Ethical Standards:}

Conflict of Interest: Dr. Hernandez, Nauman, Thomas, O'Brien, and Forrest report grants from PCORI, during the conduct of the study. Dr. Forrest reports additional grants from FDA, CDC, NIH, AHRQ, and Lily; none of these grants relates to the content of this work. Dr. O'Brien also reports grants from BMS, Novartis, and Amgen.

Disclaimer: The statements presented in this publication are solely the responsibility of the authors and do not necessarily represent the views of other organizations participating in, collaborating with, or funding PCORnet or of the Patient-Centered Outcomes Research Institute (PCORI).

\section{REFERENCES}

1. Centers for Disease Control and Prevention - National Institute of Occupational Safety and Health. Available at: https://www.cdc.gov/ niosh/topics/healthcare/default.html. Accessed on December 1, 2020.

2. Gibson DM, Greene J. Risk for severe COVID-19 illness among health care workers who work directly with patients. J Gen Intern Med 2020;35:2804-2806.

3. American Hospital Association. Hospitals and health systems face unprecedented financial pressures due to COVID-19. Available at: https://www.aha.org/guidesreports/2020-05-05-hospitals-and-healthsystems - face-un precedented-financial-pressures due\#: :text=The $\% 20$ AHA $\% 20$ estimates $\% 20$ the $\% 20$ net, treating\%20COVID\%2D19\%20patients\%20alone. Accessed on December 1, 2020.

4. Rae M, Claxton G, Kurani N, McDermott D, Cox C. Potential costs of COVID-19 treatment for people with employer coverage. Available at: https://www.healthsystemtracker.org/brief/potential-costs-of-coronavirus-treatment-for-people-with-employer-coverage/. Accessed on December $1,2020$.

5. Lai J, Ma S, Wang Y, Cai Z, Hu J, Wei N, et al. Factors associated with mental health outcomes among health care workers exposed to coronavirus disease 2019. JAMA Netw Open 2020;3(3):e203976.

6. Tan BY, Chew NW, Lee GK, Jing M, Goh Y, Yeo LL, et al. Psychological impact of the COVID-19 pandemic on health care workers in Singapore. Ann Intern Med 2020;173:317-320.

7. Chou R, Dana T, Buckley DI, Selph S, Fu R, Totten AM. Epidemiology of and risk factors for coronavirus infection in health care workers. Ann Intern Med 2020;173:120-136.

8. Jeremias A, Nguyen J, Levine J, Pollack S, Engellenner W, Thakore A, et al. Prevalence of SARS-CoV-2 infection among health care workers in a tertiary community hospital. JAMA Internal Med. Published online August 11, 2020. https://doi.org/10.1001/jamainternmed.2020.4214.

9. Moscola J, Sembajwe G, Jarrett M, Farber B, Chang T, McGinn T, et al. Prevalence of SARS-CoV-2 antibodies in health care personnel in the New York City area. JAMA. 2020;324:893-895.

10. Vahidy FS, Bernard DW, Boom ML, Drews AL, Christensen $\mathbf{P}$, Finkelstein $\mathbf{J}$, et al. Prevalence of SARS-CoV-2 infection among asymptomatic health care workers in the greater Houston, Texas, area. JAMA Netw Open 2020;3(7):e2016451.

11. Dzau VJ, Kirch D, Nasca T. Preventing a parallel pandemic - a national strategy to protect clinicians' well-being. N Engl J Med 2020;383:513-5.

12. Gross CP, Essien UR, Pasha S, Gross JR, Wang S-y, Nunez-Smith M. Racial and ethnic disparities in population-level Covid-19 mortality. J Gen Intern Med 2020;35:3097-3099.

13. Hooper MW, Nápoles AM, Pérez-Stable EJ. COVID-19 and racial/ethnic disparities. JAMA. 2020;323:2466-2467.

14. Martinez DA, Hinson JS, Klein EY, Irvin NA, Saheed M, Page KR, et al. SARS-CoV-2 positivity rate for Latinos in the Baltimore-Washington, DC region, JAMA. 2020;324:392-395.

15. Yancy CW. COVID-19 and African Americans. JAMA. 2020;323(19):1891-2.

16. Forrest CB, McTigue KM, Hernandez AF, Cohen LW, Cruz H, Haynes K, et al. PCORnet ${ }^{\circ} 2020$ : current state, accomplishments, and future directions. J Clin Epidemiol 2020;129:60-67.

17. Dolan ED, Mohr D, Lempa M, Joos S, Fihn SD, Nelson KM, et al. Using a single item to measure burnout in primary care staff: a psychometric evaluation. J Gen Intern Med 2015;30:582-7.

18. McMurray JE, Linzer M, Konrad TR, Douglas J, Shugerman R, Nelson $\mathbf{K}$. The work lives of women physicians results from the physician work life study. The SGIM Career Satisfaction Study Group. J Gen Intern Med 2000; 15:372-80.

19. Steptoe A, Deaton A, Stone AA. Subjective wellbeing, health, and ageing. Lancet. 2015;385:640-8. 
20. Shiffman S, Stone AA, Hufford MR. Ecological momentary assessment Annu Rev Clin Psychol 2008;4:1-32.

21. Gallup. Gallup 2019 Global Emotions Report. Available at: https://www. gallup.com/analytics/248906/gallup-global-emotions-report-2019.aspx. Accessed on December 1, 2020.

22. CDC COVID-19 Response Team. Characteristics of health care personnel with COVID-19-United States, February 12-April 9, 2020. MMWR Morb Mortal Wkly Rep. 2020;69:477-81.
23. United States Census Bureau. Your health care is in women's hands. Available at https://www.census.gov/library/stories/2019/08/yourhealth-care-in-womens-hands.html. Accessed December 1, 2020.

Publisher's Note: Springer Nature remains neutral with regard to jurisdictional claims in published maps and institutional affiliations. 